\title{
On the Construction Concept and Mechanism of Youth Responsibility Ethics in the Context of Globalization
}

\author{
Bingfeng Meng ${ }^{1, a}$, Manfei Cao ${ }^{2, b^{*}}$ \\ ${ }^{1}$ School of Marxism, Xi ' an University of Technology Xi'an , China 710054 \\ ${ }^{2}$ School of Marxism, Xi ' an University of Technology Xi'an , China 710054 \\ amon8217@163.com \\ b*murphy997@163.com
}

\begin{abstract}
It is the core of people ' $\mathrm{s}$ research to examine responsibility from the perspective of ethics. People should pay attention to the moral value and ethical significance of their own behavior, that is, ethical considerations and moral questioning of their responsibilities in practical activities. The construction of young people' s responsibility ethics must establish the humanistic idea. Starting from the basic point of ' people in the relationship', the construction of young people' s responsibility ethics should return to the life world itself, so as to truly eliminate the interpersonal estrangement and truly construct the concept and practice of young people 's responsibility ethics.
\end{abstract}

Keywords: adolescents; responsibility; responsibility ethics.

\section{INTRODUCTION}

Since the first industrial revolution, the continuous liberation and development of productive forces have made the economy, politics, culture and society between countries and regions move towards modernization. Globalization and information network are the two most significant characteristics of the world 's modernization. Globalization and information modernization make the relationship between countries and regions become more closely. No country and region can survive and develop independently in this context. Globalization and information networking have brought great opportunities and unprecedented challenges to China's development and progress. Teenagers as the direct experience of globalization and information network, due to the instability of their own values, the impact of both is the most profound and extensive. The wave of development in today' s world has a particularly profound impact on the sense of responsibility and mission of young people, and puts forward new tasks and challenges for the construction of contemporary youth responsibility ethics in China.

\section{THE CONSTRUCTION OF YOUTH RESPONSIBILITY ETHICS IN THE CONTEXT OF GLOBALIZATION}

As early as the 19th century, Marx pointed out the inevitable trend of the future development of the world communication stage in Communist Manifesto, which is based on globalization. Marx pointed out that, with the gradual development of productive forces and the continuous opening up of the world market:'Make the production and consumption of all countries a world... In the past, the state of self-sufficiency and self-preservation of local and ethnic groups was replaced by the mutual exchanges and interdependence of various ethnic groups. So is the production of material and spiritual'. [1]That is to say, global economic exchanges will inevitably bring comprehensive exchanges between countries and regions, which is bound to have a great impact on the construction of adolescent responsibility ethics in China.

\subsection{Opportunities for the Construction of Youth Responsibility Ethics in China under the Background of Globalization}

Globalization has an overall impact on the further development of the great cause of socialism with Chinese characteristics in the new era. For the construction of contemporary youth responsibility ethics, globalization 
has brought many favorable conditions for the cultivation and education of young people in China.

First, it broadens the vision of the construction of adolescent responsibility ethics in China. Globalization has made great changes in the cultivation mode and environment of China ' s original sense of responsibility, which has gradually changed from the original closeness to openness. The most direct and main influence of the change of the model is that it has largely changed the original old way of thinking of the construction of youth responsibility ethics. Correspondingly, globalization also makes multicultural elements in the world agitate and promote each other, and also promotes the renewal and improvement of the concept and mode of thinking in the construction of adolescent responsibility ethics in China. In this context, China has gradually begun to examine the problems existing in the development process of contemporary ideological education, focusing on reflection on the shortcomings of contemporary youth responsibility education and moral education, and reimprove the evaluation criteria of contemporary youth ideological education. All these provide a broader perspective and mode for the construction of youth responsibility ethics in China. Global cultural integration provides a broader channel and platform for contemporary youth to understand the world and society. With the help of network, video, books and other information media and carriers, they can fully and comprehensively understand the commonness and individuality of world culture and the practical problems of commonness and individuality of human society. Understanding the disharmonious factors in the world, such as ecological problems, environmental problems, terrorism, energy crisis and other global problems, these problems are not solved by any country or region, but can be resisted, prevented and solved only by the concerted cooperation between various countries. In this sense, it also provides a new thinking for the research and construction of adolescent responsibility ethics in China, and expands the research field of vision.

Second, to further update the channels and methods of adolescent responsibility ethics construction. For the cultivation and construction of adolescent responsibility ethics, all countries and regions in the world have their own content characteristics, methods, and these in the context of globalization, the construction of adolescent responsibility ethics in China has played a great role. Among them, the most representative is recessive education, practical education and other methods gradually introduced into the field of education in China, is widely adopted and absorbed by the education sector, and after practice proves that these methods are very effective. Dewey pointed out : ' This kind of good character can not only rely on personal advice, example guidance or persuasion education to achieve, but also rely on a certain organization or social life to affect individuals, so as to achieve this good character.
[2]Dewey 's view has a great influence on the construction of adolescent responsibility ethics in China. In other words, for the cultivation of adolescent responsibility ethics, we should guide adolescents to exercise themselves in practice and understand their social responsibilities in practice. It is necessary to put teenagers under a reasonable and scientific situation, so that they can form a conscious and correct social responsibility in practice, so as to realize the real goal of the construction of adolescent responsibility ethics in China.

\subsection{The Challenge of the Construction of Youth Responsibility Ethics in China under the Background of Globalization}

Materialism emphasizes that the positive and negative sides of things should be treated dialectically. Globalization has both positive and negative effects on the construction of adolescent responsibility ethics in China. We must dialectically view the impact of globalization on the construction of adolescent responsibility ethics in China.

First, the erosion of globalization on contemporary youth patriotism beliefs. The continuous disintegration and erosion of the patriotic beliefs of contemporary youth in China by globalization is a great challenge to the construction of adolescent responsibility ethics in China. Its main performance is the impact of globalization on China ' $\mathrm{s}$ youth patriotism and national consciousness. National consciousness, as an individual's identity and emotional consciousness to his nation and country, is the cornerstone for the individual to correctly establish patriotism. The weakening of national consciousness will lead to the weakening of young people' s identity to the nation and the country, love their country and the nation is a national obligation of young people is more responsibility. Therefore, with the weakening of national consciousness, it is bound to affect the continuous lack of patriotic feelings of young people in China. For the construction of young people' s responsibility ethics, this is a huge challenge that it will directly face. The characteristics of globalization, such as openness and integration, give the possibility and opportunity for the culture of all countries and regions in the world to be widely disseminated worldwide. With the help of economic advantages, western countries spread their cultural thoughts and values in large scale, which makes Chinese teenagers have a subtle influence in the process of contacting with these cultures, and gradually enhance the identity of western values. This situation is extremely unfavorable to the orderly development of Chinese culture and the healthy growth of young people. Under the influence of such culture, contemporary teenagers will gradually weaken their national consciousness and patriotism, and eventually lead to serious obstacles to the construction of adolescent responsibility ethics in China. 
Second, the impact of globalization on the traditional values of contemporary youth. With the continuous development and deepening of globalization, Western cultural thoughts and values continue to pour into China like a tidal stream, which has formed a noticeable impact on the moral cognition, value orientation and responsibility consciousness of contemporary adolescents. The most striking feature of western values is the emphasis on pragmatism, individualism and heroism, the emphasis on the realization of individual values, and the link between individualism and pragmatism and the sense of responsibility and mission of the country. This concept of individualism and pragmatism is completely opposite to the value concept of collectivism and selfless dedication advocated by Chinese tradition. Influenced by the western values, Chinese teenagers have gradually begun to have different degrees of identity with the values of individualism, pragmatism and heroism, which will bring great challenges and shocks to the construction of Chinese teenagers ' responsibility ethics.

\section{THE CONCEPTUAL PRINCIPLES OF THE CONSTRUCTION OF ADOLESCENT RESPONSIBILITY ETHICS IN THE CONTEXT OF GLOBALIZATION}

\subsection{The concept of construction}

First, the concept of ' relationship'. Marx pointed out that' human nature is not the abstract inherent in a single person, in its reality, it is the sum of all social relations. ' [3]In reality, human nature is the sum of social relations formed by human beings in social practice, and it is an important basis for distinguishing the essential characteristics of human beings and animals. Human is the carrier of social relations, in the social relations with people 's way for purposeful production and other social members of social relations, if such purpose and relationship fade, this has substantial, in the relationship of the individual will no longer exist. In today' s social transition period, great changes have taken place in the communication between people. Under the increasingly informationized social conditions, the relationship is limited to a certain regional scope. The values and responsibility concepts established with the differences of time and space reflect certain dynamics. This special construction gives the coexistence of responsibility. From the perspective of contradictions and problems in the new era, it shows that people must share risks and responsibilities, and provide the necessary life and death and common value structure for the construction of public ethics. In Marx ' $\mathrm{s}$ view, only on the premise of respecting the development of other free people in society, can people achieve their own free development by themselves. When people construct responsibility ethics, they are relatively limited by many factors. When there is no condition for taking responsibility, free development will become difficult. In other words, close social relations are an important basis for the construction of responsibility ethics. Only by constructing a stable and effective responsibility ethics can people establish harmonious social relations with other members in society and promote their own free development.

Second,' subject' concept. The reason why an individual can turn himself into a subject in an objective activity is that the individual constantly uses consciousness in life activities to influence his physiological and psychological structure, and adapts to the will brought by society and nature through various positive or negative behaviors. In this process, the individual can give full play to his subjective initiative and enhance his practical ability. When people can impose labor activities on other things, the transformed subjective ability is immeasurable. The highest form of the development of individual subject is the class subject. The distinctive characteristics of the family, the state and the society reveal the most real and obvious symbol of the individual as the subject. They exist as a relationship network or a relationship chain. They always maintain an open and inclusive attitude to carry out social dialogue, so that individuals realize that only continuous communication can realize their subjectivity and become a real social person. This is the only way to transcend the limitations of modern individual subjects. Since people can make unremitting efforts in dealing with object activities, families, countries and society, as historical subjects, will construct reasonable and effective responsibility ethics to undertake the historical mission entrusted to them by the transition period. In the current social transformation period, it is also urgent to reduce and eliminate the negative energy brought about by social transformation through individual positive production practice. As an important period of positive social transition, adolescents have to transform from dependence to autonomy, externalize and construct the behavioral pattern of individual subjectivity through" self in the mirror", and become the "mainstay " of social development after being recognized and accepted by society. Understanding and constructing the subjectivity of responsibility ethics and moral cognition has become the most critical content.

\subsection{Principles of construction}

First, the principle of public reason. Public reason from its essential characteristics, it is a kind of gradual rationality, supplemented by the effect of justice in order to promote equality of all parties, under the guidance of the political system to produce strong value logic system content. As a public ethics in public rationality, it is to try to establish a good vision for everyone to participate in the fulfillment of obligations within the society, which is a process of continuously improving citizens 
ideological and political morality. In the perspective of the principle of public reason, responsibility refers to the individual, the state and society to undertake the rights and obligations given by the society with practical action under the premise of adapting to their own roles. Public reason is public, is the exercise of public power in the public sphere, the thought, spirit, cultural concepts and other things with public nature rooted in the behavior of social people, the use of positive effects of public reason to deepen the implementation of social responsibility, make it actively fulfill their responsibilities. As a unique public existence, responsibility is an important tool for maintaining and realizing social public interests. In order to achieve public interests, it is necessary to truly promote the realization of public interests from the perspective of public rationality. In order to maintain the sustainable development and stability of our country and society, it is necessary to accelerate the construction of public rationality at all levels of society and improve the responsibility of social subjects under the guidance of public rationality. There is no doubt that public reason is a simple assumption in western philosophy, but only the guidance of the principle of public reason can make all sectors of society bear the important responsibility of building ethical system and maintaining ethical operation. In the contemporary era under the background of globalization, multiple stakeholders and multiple values coexist. For the construction of adolescent responsibility ethics, we need to reach a rational consensus that is generally accepted by all members of the society with the public interest as the purpose through respect and tolerance, equality and justice, communication and communication, so as to guide people' $\mathrm{s}$ behavior orientation and maintain the order and harmony of social public life. From the above, it can be seen that the core of public reason lies in publicity, the essence lies in public good, and the purpose is to seek public interest. It will inevitably become the key category of constructing public life and forming consensus among multiple subjects. [4] Therefore, under the guidance of the principle of public reason, it is an objective requirement for the construction of adolescent responsibility ethics to actively cultivate the tolerance spirit and responsibility consciousness of adolescent subjects and improve their moral and rational abilities.

Second, the responsibility standard principle. "Standard " means" original seat " and " original position ". At the existing academic level, it is an analytical method and research paradigm with instrumentality, which expresses the focus in the specific value goals of things. The essence of the standard of responsibility and right here means that in many relationships such as human 's responsibility, rights and obligations, responsibility and rights are the fundamental and foundation. It should take the responsibility and rights of the subject as the logical starting point, the core and the leading. What we should do is to pay equal attention to responsibility and rights, and take responsibility and rights as the reference point of all activities to connect with everything to achieve the implementation of its obligations. However, the power and responsibility standard is not equal to the individual standard, nor can it separate ' rights ' and ' responsibility '. As American scholar Mary Ann Glenton said : ' In our standard, people tend to express rights as absolute, personal, and irresponsible things, which makes the American version of the right discourse become unique. Continuing dialogues on rights and responsibilities occur in several other liberal democracies. When we examine this, the single form and overconfidence of our rights discourse are more obvious. ' This view only caters to the economic, immediate and individual dimensions of the rights issue, but it often ignores its moral, long-term and social connotations.' [5] The matching of rights and responsibilities is an important guarantee for social members to effectively fulfill their responsibilities. Therefore, an important principle for the construction of responsibility ethics is the standard of rights and responsibilities. The total size of rights and responsibilities here is that every minute and every second must have the right and responsibility. It can be seen that the principle of taking power and responsibility as the responsibility ethics construction is the inevitable requirement of public ethics. With the rapid development of society and the deepening of globalization, it is increasingly difficult for the government to rely solely on its own strength to control and manage the increasingly complex public affairs, and the assumption of individual responsibility and the construction of responsibility ethics are particularly important. Therefore, in the practice of adolescent responsibility ethics construction, adhere to the principle of rights and responsibilities is to treat the rights of each subject equally, and to respect and protect the rights of young people and all people to the maximum extent. At the same time, it emphasizes that through the coordination of relations between people, as well as moral norms, legal systems, etc., people should establish corresponding responsibilities to each other, and ultimately in the specific practice of moral life, these responsibilities and rights are gradually transformed into specific external behavior habits, so that they can take responsibility for society to establish a social cornerstone.

Third, the principle of unity of knowledge and action. The formation of responsibility ethics usually starts from the cognition of responsibility, and the recognition and practice of responsibility ethics only begin with the cognition of responsibility ethics. Therefore, responsibility cognition is the core of the formation process of adolescent responsibility ethics. First of all, responsibility cognition is the starting point and the necessary process for teenagers to form the concept of responsibility ethics, which determines the direction and depth of the development of responsibility ethics. Secondly, responsibility cognition can dominate 
individual behavior. Responsibility cognition plays a connecting role in social responsibility, which can transform scattered cognition into stable concepts and be practiced in reality. Young people' s sense of social responsibility should not only stay in the theoretical stage, more importantly, through practical activities to practice the theoretical knowledge into personal behavior. Finally, responsibility cognition and responsibility behavior are not completely equal. Sometimes, there are situations where responsibility cognition and responsibility behavior are not equal. There are differences in words and deeds, and theories and practices are not consistent. This requires us to continuously solve this contradiction and combine responsibility cognition and responsibility behavior. Young people' $s$ responsibility ethics is gradually formed in the contradiction between their responsibility cognition and responsibility behavior. Therefore, in the process of constructing youth responsibility ethics, we should pay attention to the unity of responsibility cognition and responsibility behavior. Specifically, in the process of constructing responsibility ethics, we must fully take into account the current social background and the main characteristics of China. The combination of knowledge and action needs to play a key role in the practice of responsibility, so that all people, including adolescents, understand what responsibility is, why we should construct a responsibility ethics system, how to assume and implement responsibility, and solve the problem of basic consciousness and initiative. Pay attention to social practice, strengthen the responsibility behavior training, promote the formation and transformation of the responsibility consciousness, let the youth theme not only know what is the responsibility, to take what responsibility, but also to make it effectively fulfill the responsibility, through the test of practice and exercise to develop a habit of nature, let the principle of unity of knowledge and practice in the construction of responsibility ethics deeply rooted in the hearts of the people, perseverance.

\section{THE CONSTRUCTION MECHANISM OF YOUTH RESPONSIBILITY ETHICS UNDER GLOBALIZATION}

The construction and improvement of adolescent responsibility ethics is the formation process under the influence of comprehensive factors such as internal natural evolution and external institutional environment. The specific implementation mechanism of adolescent responsibility ethics construction mainly includes the following aspects :

\section{1. leading mechanism}

Teenagers as minors, personal outlook on life values world outlook has not yet matured and stereotyped, need parents, teachers and social media guidance and guidance. All morals are a system of many rules, and the essence of all morals is that individuals learn to obey them. However, when explaining how people learn to obey the rules, they begin to diverge. [6]Piaget ' $s$ efforts to explore ' in the field of children 's psychology analysis of this ' how ' problem ' tells us : teenagers in compliance with the rules, more imitation of adult practice, it does not know the meaning of these acts, and when they begin to learn to understand why to comply with these rules, itself is a process of persuading themselves, by understanding the meaning of the behavior will be fixed, into their own code of conduct. That is to say, in the construction of adolescent responsibility ethics, we also need to guide young people to understand the meaning of behavior, let young people know what kind of behavior is reasonable, what kind of behavior is unreasonable. Needs and motivation are the premise and internal motivation of behavior. Therefore, in terms of the construction of adolescent responsibility ethics, it is necessary to form the needs and motivation of responsibility ethics in their hearts, and clarify the specific objectives of the construction of responsibility ethics. In this process, through the guidance of educational subjects, adolescents can clarify their social roles and social responsibilities, understand the behavior and emotion of different roles from different angles, and think and experience the feelings of others in order to better assume and perform their social responsibilities.

\section{2. incentive mechanism}

Incentive mechanism is a strengthening mechanism, including positive strengthening and negative strengthening. In the behavior thought of adolescent responsibility ethics, some behavior thought is correct and should be strengthened positively. Through the positive reinforcement, this behavior is standardized and fixed. Some behavior thought is wrong. It should be based on negative reinforcement and reduce this wrong behavior through negative reinforcement.

First of all, adolescents should carry out selfmotivation. Young people should recognize their psychological characteristics and practical abilities. In social life, they should neither despise themselves nor have unrealistic expectations of their abilities. Instead, they should show their actual abilities and levels, maintain psychological balance, strengthen self-efficacy and assume corresponding responsibilities with a healthy attitude. Secondly, example incentive is also a very necessary and effective means. Through the power of example, adolescents concretize their own ethical concepts of responsibility, set clear goals for their own responsible behaviors, and accordingly establish the target content and methods of their own ethical responsibility, so as to gradually complete the construction of their own ethical responsibility in a subtle way. Finally, the construction of adolescent responsibility ethics has always been a complex three- 
dimensional project, is the result of the joint action of individual subject and society, in the incentive mechanism, the society should provide positive external stimulation and incentive, so that young people can experience practice in real life, feel the joy and sense of mission of responsibility, and the lack of young people in the process of fulfilling their responsibilities should be divided into technical, psychological support and counseling, through the process and results of the dual incentive to ensure the long-term and stability of the construction of adolescent responsibility ethics.

\section{3. monitoring mechanism}

The monitoring mechanism is essentially an accountability mechanism, which contains two meanings, one is the cognition and understanding of the responsibility ethics construction activity itself, the other is to test the results of the responsibility ethics construction activity. In the process of monitoring the construction of responsibility ethics, does the educational subject have the duty to guide young people to understand and understand the meaning and connotation of responsibility ethics, whether it is to encourage the correct behavior of young people, correct the wrong behavior, and guide young people to establish their own responsibility ethics system. Then, the responsibility ethics system of adolescents is regularly tested, specifically as follows : school teachers, together with social media, regularly consider adolescents , responsibility awareness and behavior character, and judge whether their behaviors meet their responsibility ethics system and social requirements; Social media reports and promotes social models ; Schools use a variety of different methods to develop students in the process of ethical responsibility required character. In addition, it is necessary to establish the early warning mechanism of youth crisis, improve the supervision and monitoring mechanism of youth, discover the fluctuation of youth' s thought, psychology and behavior in time, prevent the emergence of youth problems in advance, and avoid the occurrence of malignant groups and sudden events. Some people have questioned the rationality of the monitoring mechanism. In fact, the academia has always believed that morality cannot be evaluated by quantitative standards. This chapter is not a quantitative measurement of the actual effect of the construction of responsibility ethics, but hopes to find and correct the problems and deviations in the construction of responsibility ethics through this method, and construct adolescent responsibility ethics.

\section{4. regulation mechanism}

The regulatory mechanism is to correctly guide the problems found in the monitoring mechanism and bring them back to the correct track. Especially for the bad behaviors and thoughts of adolescents, the regulatory mechanism is based on the conclusions drawn from the monitoring mechanism. It traces the causes of the problems, analyzes the subjective factors of adolescents, analyzes the methods of guidance and motivation, and finds out the relevant reasons. On this basis, it guides again, brings the understanding and understanding of adolescents into the range consistent with the requirements of the times, analyzes the analyzes the causes of the original problems, and guides adolescents to analyze the causes independently, re-recognize and understand, and ethics of adolescents through the gradual implementation of the rising way. First of all, it is necessary to strengthen the internal control of adolescents, externalize their internal motivation into behavior, absorb the essence of traditional responsibility ethics of the Chinese nation, and combine with external norms to cope with the transformation period of reform with an open mind. Secondly, we should strengthen the ideological control, respect the personality differences and cognitive gaps of young people, in order to cultivate young people' $\mathrm{s}$ correct cognitive ability and discrimination ability and healthy personality, in order to face the responsibility and take responsibility. Finally, attention should be paid to the regulation of practical ability. The spirit of cooperation and sense of responsibility in the construction of adolescents' responsibility ethics should eventually be transformed into action. Therefore, adolescents ' psychology and thoughts should be tested through practical activities. The problems in the process of adolescents ' practical behavior should be analyzed, and the root causes should be traced to solve the problems. The realization and self-improvement of adolescents' self-worth should be guided through feedback.

\section{CONCLUSION}

To construct adolescent responsibility ethics, primarily, seize the opportunities brought about by globalization, and cope with the challenges brought about by the rapid development of science and technology. Clarifying the concept of ' relationship ' and ' subject ', define the object and purpose of the construction of responsibility ethics, and further standardize the construction process according to the principle of public reason, the principle of responsibility-based and the principle of unity of knowledge and action. This paper also puts forward the guiding mechanism, incentive mechanism, monitoring mechanism and control mechanism, forming a complete set of concrete implementation mechanism of adolescent responsibility ethics construction.

\section{ACKNOWLEDGMENTS}

Supported by the Shaanxi Social Science Fund Project: Research on the Construction Mechanism of College Students' Moral Responsibility in the New Era (number: 2019A22). 


\section{REFERENCES}

[1] Marx, K., Engels, F. Marx \& Engels Collected Works Vol 01. People's Publishing House, Beijing. 2012. pp. 404.

[2] Dewey, J. Democracy and Education. Translated by Chengxu Wang. People's Education Press, Beijing. 2001. pp. 246.

[3] Marx, K., Engels, F. Marx \& Engels Collected Works Vol 01. People's Publishing House, Beijing. 2012. pp. 135.

[4] Bingfeng Meng, Intersubjectivity Moral Personality Education Research. Doctoral Dissertation of Xi'an University of Technology. People's Publishing House, Beijing. 2010.

[5] Glendon. M. A. Rights Talk--The Impoverishment Of Political Discourse. Translated by Wei Zhou. Peking University Press, Beijing. 2006. pp. 15, 226.

[6] Kohlberg.L. Philosophy of moral education. Translated by Xianchao Wei. Zhejiang Education Press, Hangzhou. 2000. pp. 376. 